\title{
BMJ Open Effects of individual and parental educational levels on multimorbidity classes: a register-based longitudinal study in a Danish population
}

\author{
Stine Schramm (D) , Sanne Pagh Møller (D) , Janne Schurmann Tolstrup, \\ Bjarne Laursen
}

To cite: Schramm S, Møller SP, Tolstrup JS, et al. Effects of individual and parental educational levels on multimorbidity classes: a register-based longitudinal study in a Danish population. BMJ Open 2022;12:e053274. doi:10.1136/ bmjopen-2021-053274

- Prepublication history and additional supplemental material for this paper are available online. To view these files, please visit the journal online (http://dx.doi.org/10.1136/ bmjopen-2021-053274).

Received 13 May 2021 Accepted 03 February 2022

A) Check for updates

(c) Author(s) (or their employer(s)) 2022. Re-use permitted under CC BY-NC. No commercial re-use. See rights and permissions. Published by BMJ.

National Institute of Public Health, University of Southern Denmark, Copenhagen, Denmark

Correspondence to Stine Schramm; stis@sdu.dk

\section{ABSTRACT}

Objectives To assess the effects of individual educational level in adulthood and parental educational level during childhood, as well as combinations of individual and parental educational levels, on multimorbidity classes. Design and setting In this longitudinal study, we used data from a random sample of the Danish population aged 32-56 years without multimorbidity in 2010 ( $n=102818)$. The study population was followed until 2018. Information on individual and parental educational levels and chronic conditions was obtained from national registers. Multinomial logistic regression analyses were adjusted for sex, age and ethnicity.

Outcome measure Seven multimorbidity classes were identified using latent class analysis based on 47 chronic conditions. Persons deceased during follow-up comprised a separate class.

Results We found an independent effect of individual educational level on five multimorbidity groups and death, most pronounced for the multimorbidity group 'Many conditions' (OR=1.89, 95\% $\mathrm{Cl} 1.58$ to 2.26 for medium and $\mathrm{OR}=3.22,95 \% \mathrm{Cl} 2.68$ to 3.87 for short compared with long educational level) and of parental education on four groups and death, most pronounced for the multimorbidity group 'Many conditions' ( $O R=1.36,95 \%$ $\mathrm{Cl} 1.07$ to 1.73 for medium and $\mathrm{OR}=1.48,95 \% \mathrm{Cl} 1.15$ to 1.89 for short compared with long educational level). Odds of belonging to four multimorbidity classes increased with lower combination of individual and parental educational levels, most pronounced for the multimorbidity group 'Many conditions'.

Conclusion As both individual and parental educational levels contribute to the risk of multimorbidity, it is important to address inequality throughout the life course to mitigate multimorbidity. Future studies could adopt a life course approach to investigate the mediating role of behavioural, clinical, environmental and other social factors.

\section{INTRODUCTION}

Multimorbidity, the co-occurrence of chronic conditions within the same individual, is an increasing public health challenge associated with decreased quality of life and functional ability, ${ }^{1-3}$ increased healthcare
Strengths and limitations of this study

- This study used register-based information on parental educational level in childhood as a measure of childhood socioeconomic status, thus removing the risk of recall bias which may have occurred using self-reported information.

- The study population was a large nationally representative sample of the Danish adult population, removing risk of selection bias found in cohort studies.

- Distinct multimorbidity groups were classified using latent class analysis and register-based information on a large number of chronic conditions, whereas previous studies mainly have defined multimorbidity based on a simple count of chronic conditions.

- Diagnostic information from primary care was not available in the Danish national registers, but information on redeemed prescriptions was used to capture relevant chronic conditions.

- The findings may only be generalised to settings with similar social and educational systems.

utilisation $^{45}$ and increased risk of mortality. ${ }^{6}$ Social inequality in multimorbidity has been reported, whereby individuals of low socioeconomic status have a higher likelihood of having multimorbidity compared with individuals of higher socioeconomic status. It is important to understand the drivers for these inequalities. A range of different mechanisms and factors can explain the inequality in multimorbidity, including but not limited to behavioural factors (such as smoking, alcohol intake, diet and physical activity), psychosocial factors (such as stress), access and utilisation of healthcare services, as well as biological and environmental factors. Additionally, there is an increasing attention towards how exposures during early life affect health and disease later in life. It is possible that socioeconomic factors during childhood contribute to the development of multimorbidity in later life, partially or fully 
mediated through adult socioeconomic status. Previous studies examining the association between childhood socioeconomic status and adult multimorbidity have shown conflicting results. ${ }^{7-13}$ Most studies relied on selfreported (retrospective recall) information about childhood conditions, ${ }^{78101213}$ which may be subject to recall bias, whereby individuals with poor current health more readily recall poor conditions in childhood. By using an objective measure such as register-based information on parental education we can elucidate this limitation.

Previous longitudinal studies on individual socioeconomic status and multimorbidity have mainly classified multimorbidity as a count of any chronic conditions, typically two or more conditions. This definition assumes that the relationship between socioeconomic status and multimorbidity is constant across levels and types of multimorbidity (ie, the same for individuals with diabetes and heart disease as for individuals with asthma and allergies). However, cross-sectional studies with specific multimorbidity groups, such as clusters of chronic conditions, have shown that the association between socioeconomic status and multimorbidity indeed varies across multimorbidity groups. ${ }^{114}$

We attempt to address the above gaps by using registerbased information on parental education as a marker for childhood socioeconomic status, highest educational level completed in adult life and multimorbidity classes based on 47 chronic conditions. The aim of the study was to assess the effects of individual and parental educational levels, as well as combinations of individual and parental educational levels, on multimorbidity classes.

\section{METHODS}

\section{Study population}

The study population was drawn from individuals invited to participate in the Danish National Health Survey (DNHS) 2010, which is a nationally representative sample of the Danish adult population that has been described elsewhere. ${ }^{15}$ For this study, we included individuals invited to participate in the DNHS, aged $32-56$ years by 1 January 2010 (40-64 years in 2018) and who did not have multimorbidity in 2010 , that is, had only one or no chronic condition at baseline $(\mathrm{n}=102818)$. The study population was linked with national registers using the unique personal identification number available to all Danish residents and followed for 8 years. Information on sex, age and ethnicity was obtained from the Danish Civil Registration System. ${ }^{16}$

\section{Patient and public involvement}

Patients and/or the public were not involved in the design, conduct, reporting or dissemination plans of this research.

\section{Educational level}

The Danish education system consists of 10 years of compulsory education in primary and lower secondary schools. However, before 1975, compulsory education consisted of 7 years. The upper secondary education system includes a range of optional programmes, where academic programmes allow students to apply for entry to higher education and vocational programmes are aimed at direct entry to the labour market. Education is open to all residents and generally free of charge. In this study, information on educational level was obtained from the Population Education Registers. ${ }^{17}$ Educational level was defined as the highest level completed and categorised as short (primary and lower secondary schools), medium (gymnasium, vocational schools, short-cycle higher education) and long (university college, university undergraduate and graduate programmes) education. This definition was applied for both individual and parental educational levels. Individual educational level was measured in year 2010, whereas parental education was measured when the individuals were 5 years of age or in year 1981. Data from the Population Education Registers used in this study are only available from year 1981 and onwards. Therefore, parental educational level in childhood was measured in 1981 for individuals born before year 1976, and at age 5 years for individuals born in year 1976 or later. Information on individual and parental educational levels was missing for $2.1 \%$ and $15.4 \%$ of the total study population, respectively. Missing observations for education were included in the category short education as individuals with missing information on education in a Danish context typically are older and from a generation where long educational level was not common. ${ }^{17}$ Additionally, we conducted a sensitivity analysis with missing observations in a separate category. Here the effect estimates were similar to the estimates of short educational level (data not shown).

\section{Multimorbidity}

The outcome, multimorbidity classes, was assessed in 2018 using register-based information on 47 chronic conditions. We used algorithms applied in our previously published study. ${ }^{14}$ The included conditions and classifications are specified in online supplemental table S1. International Classification of Diseases (ICD-10) codes were obtained from the Danish National Patient Register and the Danish National Psychiatry Central Research Register, which contains information on all contacts to secondary healthcare in Denmark, as well as psychiatric hospitals and psychiatric departments. ${ }^{18} 19$ Anatomical Therapeutic Chemical (ATC) and indication codes for redeemed prescription drugs sold in Danish pharmacies were obtained from the Danish National Prescription Registry. ${ }^{20}$ We used latent class analysis (LCA) to group classes of chronic conditions that describe the multimorbidity pattern of this study population.

\section{Statistical analysis}

LCA was chosen over count of chronic conditions (ie, two or more chronic conditions) to explore the associations of educational level with multimorbidity by different chronic 
condition patterns. Furthermore, we chose LCA because the method does not entail a priori assumptions about the patterns of chronic conditions. The LCA was conducted using the 47 chronic conditions as observed indicators, with no covariates included. The optimal number of latent classes was based on the following criteria: model information-based criteria (lowest Akaike information criterion (AIC) and Bayesian information criterion (BIC)), and the smallest class included at least $1 \%$ of the total study population. Additionally, we assessed the presence of distinct classes and meaningful interpretation of each class. Models from 2 to 9 classes were evaluated. The $\mathrm{AIC}$ and $\mathrm{BIC}$ values continued to decrease as more latent classes were added to the model, but models where the smallest class included at least $1 \%$ of the total study population were prioritised (online supplemental table S2). In models with classes less than $1 \%$ of the total population, some classes did not have meaningful interpretation such as presence of only one chronic condition. The AIC and $\mathrm{BIC}$ values, class size and entropy are presented in online supplemental table S2. Each individual was allocated the classes according to the estimated probability.

To examine the association of individual and parental educational levels with multimorbidity latent classes, multinomial logistic regression using individual and parental educational levels as independent variables was used to calculate the ORs and 95\% CIs. The LCA models the probability of each individual belonging to different latent classes, where bias may be introduced from assigning individuals to the class with the highest probability. Consequently, we included weights based on the probability of belonging to this class in the multinomial logistic regression. The class 'Few or no chronic conditions' and long educational level were used as reference groups. All models were adjusted for sex (male; female), age categories (40-44; 45-49; 50-54; 55-59; 60-63 years of age) and ethnicity (Danish born; other than Danish born). Individual and parental educational levels were both examined separately and adjusted for each other. Lastly, we examined the associations of combinations of individual and parental educational levels with multimorbidity latent classes. There was no evidence of sex differences in associations of individual and parental educational levels with multimorbidity classes (all $\mathrm{p}>0.05$ for interaction and similar ORs). We therefore combined men and women in the analysis. Analyses were performed in SAS V.9.4 (SAS Institute). The SAS procedure PROC LCA was used for the latent class estimation. ${ }^{21}$

\section{RESULTS}

Table 1 shows the characteristics of the study population. About half of the study population were men (51.7\%) and 90.8\% were ethnic Danes. Long and medium educational levels were more common for the individual educational level $(27.8 \%$ and $50.9 \%$, respectively) than for parental educational level (15.8\% and $37.8 \%$, respectively). Almost half $(46.4 \%)$ of the parents had short educational level as
Table 1 Characteristics of study population $(n=102818)$

$\mathrm{n}(\%)$

\begin{tabular}{|cc}
\hline Gender & $53132(51.7)$ \\
\hline Men & $49686(48.3)$ \\
\hline Women & \\
\hline Age groups in 2018 (years) & $18714(18.2)$ \\
\hline $40-44$ & $21473(20.9)$ \\
\hline $45-49$ & $24647(24.0)$ \\
\hline $50-54$ & $21771(21.2)$ \\
\hline $55-59$ & $16213(15.8)$ \\
\hline $60-63$ & $93355(90.8)$ \\
\hline Ethnicity & $9463(9.2)$ \\
\hline Danish & \\
\hline Other than Danish & $28558(27.8)$ \\
\hline Individual education & $52323(50.9)$ \\
\hline Long & $21937(21.3)$ \\
\hline Medium & $16238(15.8)$ \\
\hline Short & $38857(37.8)$ \\
\hline Parental education & $47723(46.4)$ \\
\hline Long & \\
\hline Medium & \\
\hline Short & \\
\hline
\end{tabular}

Individual educational level in adult life measured in 2010. Parental education measured when individuals were 5 years of age or in 1981. Missing observations included in "Short education"

the highest completed educational level, compared with $21.3 \%$ of the study population (table 1 ).

The LCA resulted in a model with 7 latent classes that were named according to the chronic conditions identified as particularly prevalent in one class compared with the average of the study population and to other latent classes: 'Few or no chronic conditions' (68.3\%); 'Heart diseases, hypertension, high cholesterol' (6.1\%); 'Musculoskeletal conditions' (11.0\%); 'Diabetes, hypertension, high cholesterol' (5.7\%); 'Asthma; allergy' (2.8\%); 'Psychiatric conditions, epilepsy' (8.9.\%) and 'Many conditions' (1.5\%). The class 'Many conditions' is characterised by high prevalence of several both physical and mental chronic conditions (online supplemental table S3). Individuals who had passed away during the follow-up period were allocated to a separate group; 'Death' $(1.7 \%)$. In the following text, the classes will be referred to as 'Few', 'Heart', 'Musculoskeletal', 'Diabetes', 'Asthma, allergy', 'Psychiatric', 'Many' and 'Death', respectively. It should be noted that not all individuals in each class have the conditions of the class name, and that several other conditions can be present in each class. Online supplemental table S3 shows the prevalence of each chronic condition in the latent classes.

Table 2 shows the ORs for belonging to the different multimorbidity classes, as well as for death, according to individual and parental educational levels. For all multimorbidity classes, except for 'Asthma, allergy', increasing 


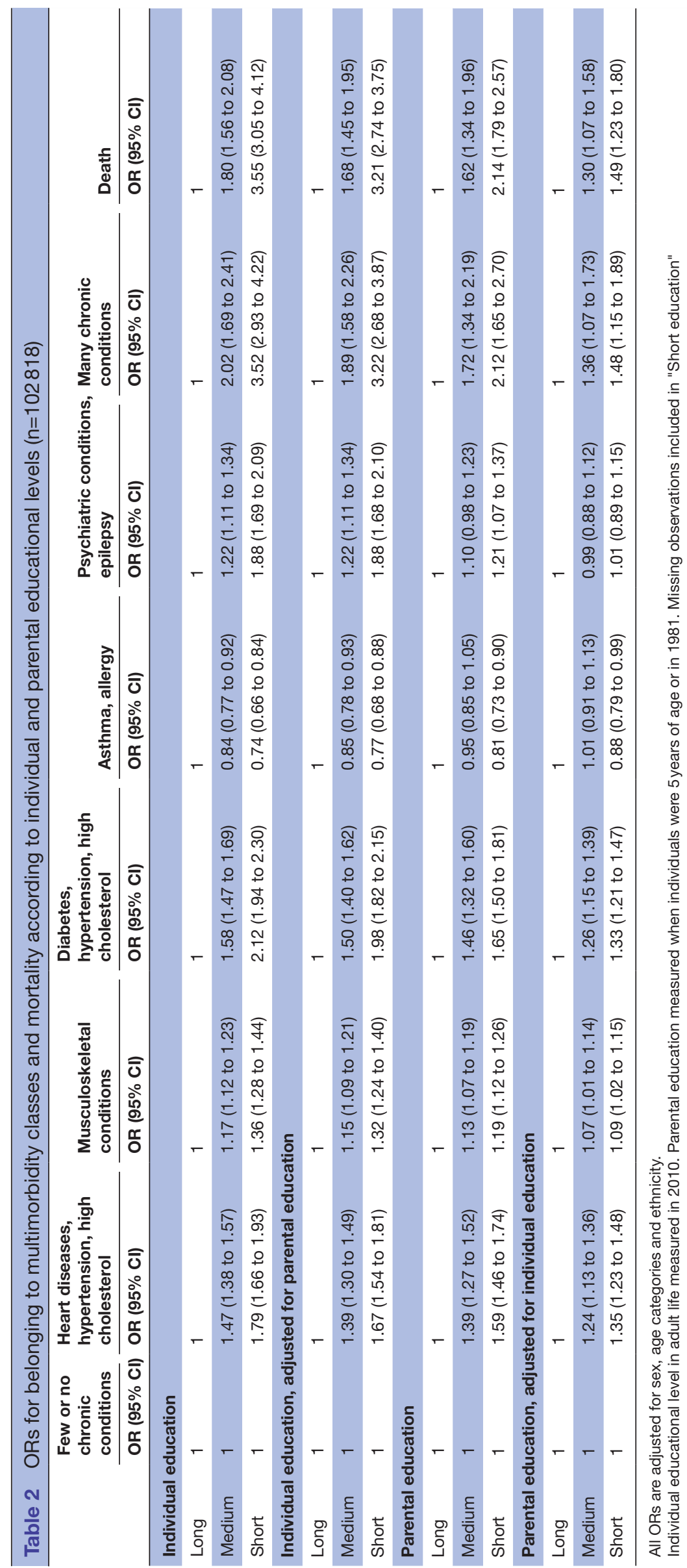

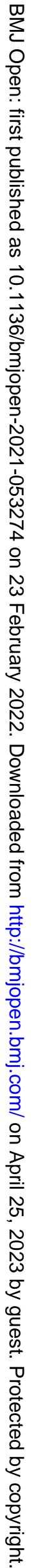




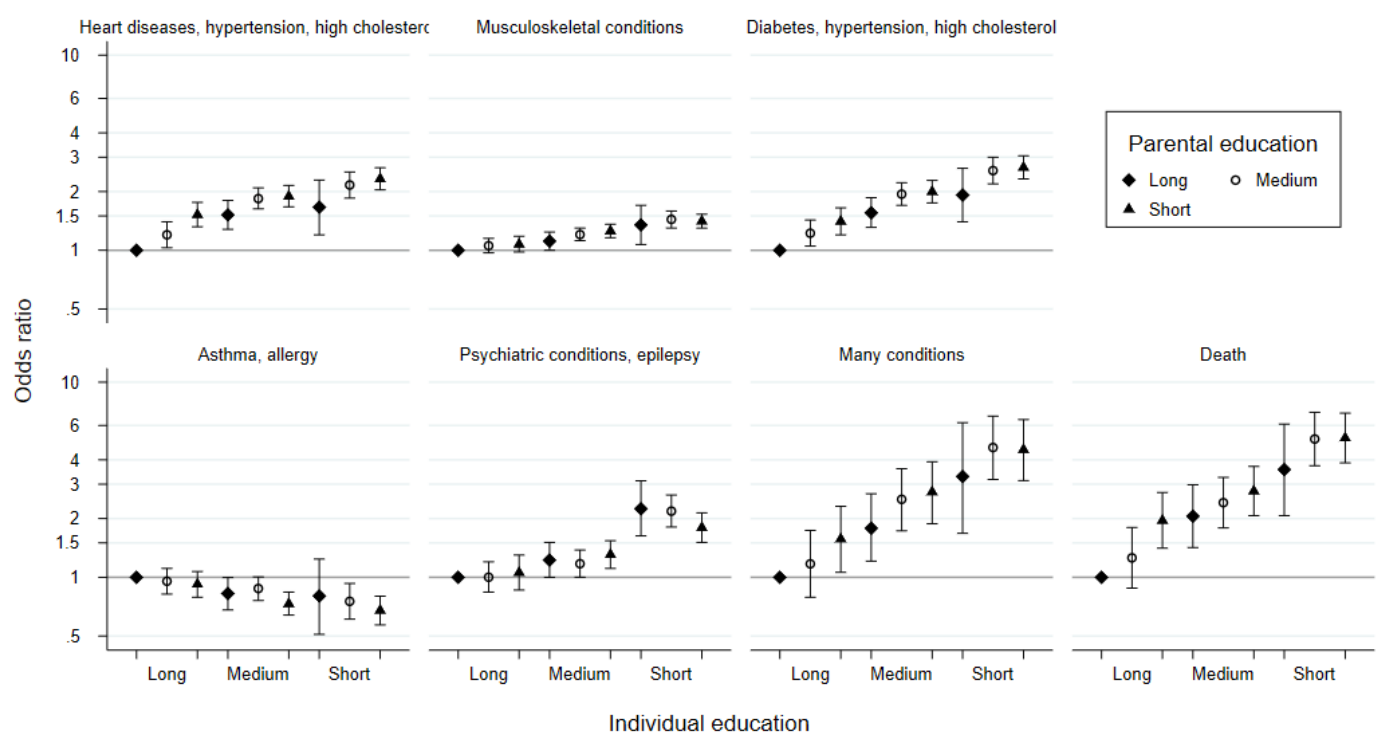

Figure 1 ORs for belonging to multimorbidity classes and mortality according to combination of individual and parental educational levels $(n=102818)$. All ORs are adjusted for sex, age categories and ethnicity. Reference group: 'Few or no chronic conditions' (not illustrated), long individual and long parental education.

odds of belonging to a multimorbidity class by lower individual educational level was seen. This was most pronounced for the class 'Many'; compared with individuals with high educational level, individuals with medium educational level had 2.02 (95\% CI 1.69 to 2.41) times higher odds, and individuals with short educational level had 3.52 (95\% CI 2.93 to 4.22) times higher odds for belonging to the class 'Many'. A similar pattern was seen for mortality. When adjusting for parental educational level, the associations remained with minimal changes (table 2).

The ORs for belonging to the multimorbidity classes according to parental educational level were similar, as with individual educational level, though with lower effect estimates and less steep increasing odds of belonging to a multimorbidity class with lower parental educational level. This was seen for all classes, except from 'Asthma, allergy', and was mostly pronounced for the class 'Many' (OR for medium parental educational level: 1.72 (95\% CI 1.34 to 2.19); and OR for short parental educational level: 2.12 (95\% CI 1.65 to 2.70$)$ ). When adjusting for individual educational level, the effect estimates were less strong and attenuated fully for 'Psychiatric'. However, associations between parental educational level and multimorbidity classes remained significant for the remaining classes (table 2).

Figure 1 shows the ORs for belonging to the multimorbidity classes, and for 'Death', by combinations of individual and parental educational levels. With high individual educational level and high parental educational level as the reference group, the ORs for combined individual and parental educational levels increased with lower level of education for the multimorbidity classes 'Heart', 'Musculoskeletal', 'Diabetes', 'Many' and for
'Death'. This is most pronounced for the class 'Many' and not seen for 'Asthma, allergy' or 'Psychiatric' (figure 1).

\section{DISCUSSION}

In this study, we found increasing odds of belonging to a multimorbidity class by both lower individual and parental educational levels, also when mutually adjusted, for the classes 'Heart', 'Musculoskeletal', 'Diabetes', 'Many' and 'Death', as well as for 'Psychiatric' for individual educational level. There was an inverse association between combinations of individual and parental education and odds of belonging to these classes. The highest odds were seen for short individual and short parental educational levels, compared with long individual and long parental educational levels. The associations were most pronounced for the class 'Many'.

Previous studies are not directly comparable in terms of measures of socioeconomic status and multimorbidity, study population, data sources and study design. However, our results on individual educational level in adulthood and multimorbidity are in line with previous literature on social inequality in multimorbidity, ${ }^{22} 23$ as well as studies using specific multimorbidity groups. ${ }^{14} 24$ Yet, some recent longitudinal studies from Canada and England found no association between education and multimorbidity, but reported that income and wealth indicators were stronger indicators when measuring social inequality in multimorbidity. ${ }^{25-27}$ In this study, educational level was chosen as a marker for socioeconomic status over other socioeconomic indicators because education is a more stable indicator over time. Income and occupation may be affected by illness and early symptoms of disease, and thus increase the risk of reverse causation when we only 
have one point in time where socioeconomic status is measured. Additionally, educational level captures the components of both income and health literacy, which may be preferred in the context of generally free access to healthcare. Findings from a large multicohort study suggest that the apparent education and wealth inequalities in adult life reflect a cumulative disadvantage due to low education and wealth in early life stages, leading to persistent differences throughout older age, with no further increase in inequality after age 70 years. ${ }^{28}$

Two previous studies used register-based information on childhood socioeconomic status, measured as father's occupation at birth ${ }^{11}$ and paternal social class. ${ }^{9}$ In line with our results, Johnston and colleagues ${ }^{11}$ found a significant association between father's occupation at birth and multimorbidity, measured as two or more conditions, after adjusting for adult educational level, whereas the association was fully attenuated in the study by Humphreys and colleagues. ${ }^{9}$ However, Humphreys and colleagues additionally adjusted for behavioural factors which may explain the full attenuation. Both studies used self-reported information on chronic conditions from cohort studies and did not distinguish between different multimorbidity groups. ${ }^{911}$

We did not find any association between individual educational level and the multimorbidity class 'Asthma, allergy'. This is in line with findings from Larsen and colleagues $^{1}$ and illustrates the importance of distinguishing specific multimorbidity groups rather than using a count of chronic conditions. For the 'Psychiatric' class, we observed increasing odds for belonging to 'Psychiatric' with lower individual educational level. However, there was no association between parental educational level and 'Psychiatric' after adjusting for individual educational level. The results on individual and parental education combinations showed that high parental educational level and short individual educational level had the highest odds of 'Psychiatric' multimorbidity. We have not come across such finding before. As we excluded individuals with multimorbidity at baseline, the risk of reverse causality should be reduced. However, we cannot eliminate the risk of late diagnosis for some individuals. Using Danish register-based information, Hakulinen and colleagues previously showed that parental income during childhood was associated with increased risk of developing a mental disorder as a young adult. ${ }^{29}$ Similar patterns were seen across a broad range of mental disorders, except for eating disorders. These findings are in line with our results on associations between parental educational level and belonging to the class 'Psychiatric' unadjusted for individual educational level in adulthood. Hakulinen and colleagues did not adjust for individual socioeconomic position in adult life..$^{29} \mathrm{In}$ the study by Hakulinen and colleagues, the associations were strongest for substance misuse and personality disorders and weaker for mood disorders and anxiety/ somatoform disorders. ${ }^{29}$ In this study, the LCA grouped the observations with a psychiatric diagnosis primarily in the same class, 'Psychiatric', and the class 'Many'. Associations between educational level and single diagnosis for chronic conditions were not examined in this study, as the focus was on multimorbidity classes.

Our study examines the overall effect of education on developing multimorbidity. There are a number of possible mechanisms that can explain our findings. First, individuals with lower educational level may be more likely to smoke, be less physically active and have a poor diet along with other unfavourable health-related behaviours, which are factors that are also correlated to a range of chronic conditions. ${ }^{30}$ Additionally, individuals with lower educational level may be more likely to live in poorer quality housing and have occupations that increase both physical and psychological stress. ${ }^{31}$ Hence, behavioural and environmental factors are likely to explain part of the social inequality in multimorbidity observed in this study. The significant associations between childhood socioeconomic status (measured as parental education) and multimorbidity classes in adult life, after adjustment for individual education, can reflect accumulative unfavourable circumstances and behaviour over the life course. ${ }^{32}$ However, genetic factors may also play a role. ${ }^{33}$ Socioeconomic circumstances in childhood and throughout the life course have shown to affect adult health outcomes, for example, for cardiovascular disease risk. ${ }^{34}$ Yet, diseasespecific models (ie, associations between socioeconomic status and specific diseases) may underestimate the true association between social and behavioural exposures, and multimorbidity, and thus the mechanisms by which socioeconomic inequalities are generated need further investigation. Future studies could focus on life course models specifying how timing in relation to social mobility, behavioural transitions, etc, influence adult multimorbidity in different ways, and additionally, by how these temporal processes are interconnected and manifested in population-level disease trends. ${ }^{35} 36$

\section{Strengths and limitations}

Our study has a number of strengths and limitations that should be considered when interpreting the findings. First, our study uses register-based information to measure childhood socioeconomic status, thus removing the risk of recall bias which may have occurred using self-reported information. The use of longitudinal design means that the measurement of education occurred before the outcome, providing support for a causal relationship. Our study uses a large nationally representative sample of the Danish adult population, removing risk of selection bias found in cohort studies. However, since educational level and multimorbidity are highly correlated, excluding individuals with multimorbidity at baseline could have resulted in a selected population of higher educational level than for the general Danish population in the applied age group. Excluding individuals with multimorbidity at baseline was a key priority to come closer to a causal estimation of the relationship between educational level and development of multimorbidity. We used register-based 
information for a large number of chronic conditions (47) to classify multimorbidity. Only one previous study examining parental education and multimorbidity used register-based information on chronic conditions. ${ }^{8}$ Unfortunately, diagnostic information from primary care is not available in the national registers. Thus, individuals who are treated for their chronic condition in general practice only will not be captured. We have used previously developed algorithms for the prescription registry using ATC and indication codes to capture these conditions, which should allow for at least partly detection of some of these diseases, but the validity of this information for identifying diseases is probably lower than that of ICD-10 codes. Lastly, our findings may only be generalised to settings with similar social and educational systems and populations of same age and other sociodemographic characteristics.

There are a number of implications to our findings. Multimorbidity and social inequality in health outcomes are increasing public health challenges. Understanding multimorbidity determinants across the life course is important to address its impact. The study illustrates the importance of addressing social inequality throughout the life course, and thus measures that mitigating the impacts of low socioeconomic status in early life and in adulthood is crucial. As the associations between educational level and multimorbidity classes differed by class, using more detailed classification of multimorbidity rather than count of chronic condition would be advantageous for future studies and programmes.

In summary, we found independent effects of individual and parental educational levels for some multimorbidity classes, but not all. Increasing odds of multimorbidity was seen for lower individual and parental educational levels for four multimorbidity groups. The association was most pronounced for a multimorbidity group with high prevalence of several both physical and mental chronic conditions. Future studies could adopt a life course approach to investigate the mediating role of behavioural, clinical, environmental and other social factors.

Contributors All authors contributed to study hypothesis, concept and design. BL did the statistical analysis with contribution from SS. All authors contributed to interpreting the data. SS wrote the first draft and successive drafts of the manuscript. SPM, BL and JST critically reviewed and edited the manuscript. SS, SPM and BL had full access to anonymised individual-level data. All authors have seen and approved the final text. SS is responsible for the overall content as guaranto.

Funding The authors have not declared a specific grant for this research from any funding agency in the public, commercial or not-for-profit sectors.

Competing interests None declared.

Patient consent for publication Not applicable.

Ethics approval Ethical approval was not necessary for this study as all information was based on national registers.

Provenance and peer review Not commissioned; externally peer reviewed.

Data availability statement Data may be obtained from a third party and are not publicly available. Due to Danish law, the confidential healthcare data used in this study can only be accessed through Statistics Denmark. Access is granted upon request to applicants who fulfil the necessary criteria. Data access requests can be sent directly to Statistics Denmark via the following email address: databanker@ dst.dk.

Supplemental material This content has been supplied by the author(s). It has not been vetted by BMJ Publishing Group Limited (BMJ) and may not have been peer-reviewed. Any opinions or recommendations discussed are solely those of the author(s) and are not endorsed by BMJ. BMJ disclaims all liability and responsibility arising from any reliance placed on the content. Where the content includes any translated material, BMJ does not warrant the accuracy and reliability of the translations (including but not limited to local regulations, clinical guidelines, terminology, drug names and drug dosages), and is not responsible for any error and/or omissions arising from translation and adaptation or otherwise.

Open access This is an open access article distributed in accordance with the Creative Commons Attribution Non Commercial (CC BY-NC 4.0) license, which permits others to distribute, remix, adapt, build upon this work non-commercially, and license their derivative works on different terms, provided the original work is properly cited, appropriate credit is given, any changes made indicated, and the use is non-commercial. See: http://creativecommons.org/licenses/by-nc/4.0/.

\section{ORCID iDs}

Stine Schramm http://orcid.org/0000-0001-8039-8095

Sanne Pagh Møller http://orcid.org/0000-0001-7552-1212

\section{REFERENCES}

1 Larsen FB, Pedersen MH, Friis K, et al. A latent class analysis of multimorbidity and the relationship to socio-demographic factors and health-related quality of life. A national population-based study of 162,283 Danish adults. PLoS One 2017;12:e0169426.

2 Kanesarajah J, Waller M, Whitty JA, et al. Multimorbidity and quality of life at mid-life: a systematic review of general population studies. Maturitas 2018;109:53-62.

3 Ryan A, Wallace E, O'Hara P, O'Hara P, et al. Multimorbidity and functional decline in community-dwelling adults: a systematic review. Health Qual Life Outcomes 2015;13:168.

4 Frølich A, Ghith N, Schiøtz M. Multimorbidity and healthcare utilization: a register-based study in Denmark. PLoS One 2019;14:e024183.

5 Palladino R, Tayu Lee J, Ashworth M, et al. Associations between multimorbidity, healthcare utilisation and health status: evidence from 16 European countries. Age Ageing 2016;45:431-5.

6 Nunes BP, Flores TR, Mielke Gl, et al. Multimorbidity and mortality in older adults: a systematic review and meta-analysis. Arch Gerontol Geriatr 2016;67:130-8.

7 Pavela G, Latham K. Childhood conditions and multimorbidity among older adults. J Gerontol B Psychol Sci Soc Sci 2016;71:889-901.

8 Dekhtyar S, Vetrano DL, Marengoni A, et al. Association between speed of multimorbidity accumulation in old age and life experiences: a cohort study. Am J Epidemiol 2019;188:1627-36.

9 Humphreys J, Jameson K, Cooper C, et al. Early-Life predictors of future multi-morbidity: results from the Hertfordshire cohort. Age Ageing 2018;47:474-8.

10 Henchoz Y, Seematter-Bagnoud L, Nanchen D, et al. Childhood adversity: a gateway to multimorbidity in older age? Arch Gerontol Geriatr 2019;80:31-7.

11 Johnston MC, Black C, Mercer SW, et al. Impact of educational attainment on the association between social class at birth and multimorbidity in middle age in the Aberdeen children of the $1950 \mathrm{~s}$ cohort study. BMJ Open 2019;9:e024048.

12 Tucker-Seeley RD, Li Y, Sorensen G, et al. Lifecourse socioeconomic circumstances and multimorbidity among older adults. BMC Public Health 2011;11:313.

13 Tomasdottir MO, Sigurdsson JA, Petursson H, et al. Self reported childhood difficulties, adult multimorbidity and allostatic load. A cross-sectional analysis of the Norwegian HUNT study. PLoS One 2015;10:e0130591.

14 Møller SP, Laursen B, Johannesen CK, et al. Patterns of multimorbidity and demographic profile of latent classes in a Danish population-A register-based study. PLoS One 2020;15:e0237375.

15 Christensen Al, Ekholm O, Glümer C, et al. The Danish National health survey 2010. study design and respondent characteristics. Scand J Public Health 2012;40:391-7.

16 Pedersen CB. The Danish civil registration system. Scand J Public Health 2011;39:22-5.

17 Jensen VM, Rasmussen AW. Danish education registers. Scand J Public Health 2011;39:91-4. 
18 Lynge E, Sandegaard JL, Rebolj M. The Danish national patient register. Scand J Public Health 2011;39:30-3.

19 Mors O, Perto GP, Mortensen PB. The Danish psychiatric central research register. Scand J Public Health 2011;39:54-7.

20 Kildemoes HW, Sørensen HT, Hallas J. The Danish national prescription registry. Scand J Public Health 2011;39:38-41.

21 Lanza ST, Dziak JJ, Huang L. Proc LCA \& Proc LTA users' guide (Version 1.3. 2). J Penn State: University Park, PA: the Methodology Center, 2015. Available: https://www.methodology.psu.edu/files/ 2019/03/proc_Ica_Ita_1-3-2-1_users_guide-2ggq4d3.pdf

22 Violan C, Foguet-Boreu Q, Flores-Mateo G, Salisbury C, et al. Prevalence, determinants and patterns of multimorbidity in primary care: a systematic review of observational studies. PLoS One 2014;9:e102149.

23 Xu X, Mishra GD, Jones M. Evidence on multimorbidity from definition to intervention: an overview of systematic reviews. Ageing Res Rev 2017;37:53-68.

24 Jackson CA, Dobson AJ, Tooth LR, et al. Lifestyle and socioeconomic determinants of multimorbidity patterns among midaged women: a longitudinal study. PLoS One 2016;11:e0156804.

25 Mounce LTA, Campbell JL, Henley WE, et al. Predicting incident multimorbidity. Ann Fam Med 2018;16:322-9.

26 Canizares M, Hogg-Johnson S, Gignac MAM, et al. Increasing trajectories of multimorbidity over time: birth cohort differences and the role of changes in obesity and income. J Gerontol B Psychol Sci Soc Sci 2018;73:1303-14.

27 Dugravot A, Fayosse A, Dumurgier J, et al. Social inequalities in multimorbidity, frailty, disability, and transitions to mortality: a 24year follow-up of the Whitehall II cohort study. Lancet Public Health 2020;5:e42-50.

28 Wu Y-T, Daskalopoulou C, Muniz Terrera G, et al. Education and wealth inequalities in healthy ageing in eight harmonised cohorts in the ATHLOS Consortium: a population-based study. Lancet Public Health 2020;5:e386-94.

29 Hakulinen C, Mok PLH, Horsdal HT, et al. Parental income as a marker for socioeconomic position during childhood and later risk of developing a secondary care-diagnosed mental disorder examined across the full diagnostic spectrum: a national cohort study. BMC Med 2020;18:323.

30 Nordahl H, Lange T, Osler M, et al. Education and causespecific mortality: the mediating role of differential exposure and vulnerability to behavioral risk factors. Epidemiology 2014;25:389-96.

31 Braveman P, Gottlieb L. The social determinants of health: it's time to consider the causes of the causes. Public Health Rep 2014;129 Suppl 2:19-31.

32 Galobardes B, Lynch J, Smith GD. Measuring socioeconomic position in health research. Br Med Bull 2007;81-82:21-37.

33 Dong G, Feng J, Sun F, et al. A global overview of genetically interpretable multimorbidities among common diseases in the UK Biobank. Genome Med 2021;13:110.

34 Lawlor DA, Ebrahim S, Davey Smith G. Adverse socioeconomic position across the lifecourse increases coronary heart disease risk cumulatively: findings from the British women's heart and health study. J Epidemiol Community Health 2005;59:785-93.

35 Kivimäki M, Batty GD, Pentti J, et al. Association between socioeconomic status and the development of mental and physical health conditions in adulthood: a multi-cohort study. Lancet Public Health 2020;5:e140-9.

36 Batty GD, Kivimäki M. Adverse childhood experiences and adult health: the need for stronger study designs to evaluate impact. $J$ Epidemiol Community Health 202110.1136/jech-2020-215870. [Epub ahead of print: 25 Jan 2021]. 